\title{
Pathophysiology, classification, and complications of common asymptomatic thrombocytosis in newborn infants
}

Ga Won Jeon, MD, PhD

Department of Pediatrics, Inha University Hospital, Inha University College of Medicine, Incheon, Korea

We frequently encounter newborn infants with thrombocytosis in the neonatal intensive care unit. However, neonatal throm. bocytosis is not yet fully understood. Thrombocytosis is more frequently identified in newborns and young infants, notably more often in those younger than 2 years than in older children or adults. The production of megakaryocytes (megakaryopoiesis) and platelets (thrombopoiesis) is mainly regulated by thrombopoietin (TPO). Increased TPO levels during infection or inflammation can stimulate megakaryopoiesis, resulting in thrombopoiesis. ТPO concentrations are higher in newborn infants than in adults. Levels increase after birth, peak on the second day after birth, and start decreasing at 1 month of age. Initial platelet counts at birth increase with gestational age. Thus, preterm infants have lower initial platelet counts at birth than late-preterm or term infants. Postnatal thrombocytosis is more frequently observed in preterm infants than in term infants. A high TPO concentration and low TPO receptor expression on platelets leading to elevated plasma-free TPO, increased sensitivity of megakaryocyte precursor cells to TPO, a decreased red blood cell count, and immaturity of platelet regulation are speculated to induce thrombocytosis in preterm infants. Thrombocytosis in newborn infants is considered a reactive process (secondary thrombocytosis) following infection, acute/chronic inflammation, or anemia. Thrombocytosis in newborn infants is benign, resolves spontaneously, and, unlike in adults, is rarely associated with hemorrhagic and thromboembolic complications.

Key words: Newborn infant, Premature infant, Platelets, Thrombocytosis, Thrombopoietin

\section{Key message}

- Thrombocytosis, common in newborns and infants ( $<2$ years) $(3 \%-13 \%)$, is caused by elevated thrombopoietin (TPO) concentrations.

- Serum TPO levels are significantly higher immediately to 1 month postnatal and decrease with age.
- Platelet counts are positively correlated with gestational age at birth and postnatal age.

- Thrombocytosis is more common in preterm than in term infants.

- Thrombocytosis in newborns is reactive and resolves spontaneously without complications.

\section{Introduction}

Thrombocytosis is known to increase the risk of hemorrhagic and thromboembolic complications in adults for which antiplatelet or cytoreductive treatment is required. ${ }^{1,2)}$ Thrombocytosis frequently occurs in newborns and young infants younger than 2 years, with an estimated incidence of 3\%-13\%, ${ }^{3)}$ although the exact prevalence is unknown. Thrombocytosis in newborn infants is considered a reactive process in most cases, and it is rarely associated with complications. ${ }^{4)}$ Whether similar risks such as hemorrhagic and thromboembolic complications in adults exist in newborn infants with thrombocytosis remains unclear. Reasons for frequent reactive thrombocytosis in newborn infants include higher levels of thrombopoietin (TPO) and more sensitive megakaryocyte precursor cells to TPO.5)

Although thrombocytosis is frequently encountered in newborn infants, especially preterm infants admitted to neonatal intensive care units, few studies have evaluated neonatal thrombocytosis. Therefore, this review aimed to evaluate the pathophy. siology, classification, and complications of thromboytosis in newborn infants. Neonatal thrombocytosis according to gestational age at birth and postnatal age was also evaluated.

\section{Pathophysiology}

\section{Megakaryopoiesis and thrombopoiesis}

Little is known about the pathophysiology underlying thrombocytosis in neonates. Platelets are nonnucleated fragments arising from megakaryocytes. Hematopoiesis transitions from

Corresponding author: Ga Won Jeon, MD, PhD. Department of Pediatrics, Inha University Hospital, Inha University College of Medicine, 27 Inhang-ro, Jung-gu, Incheon 22332, Korea 


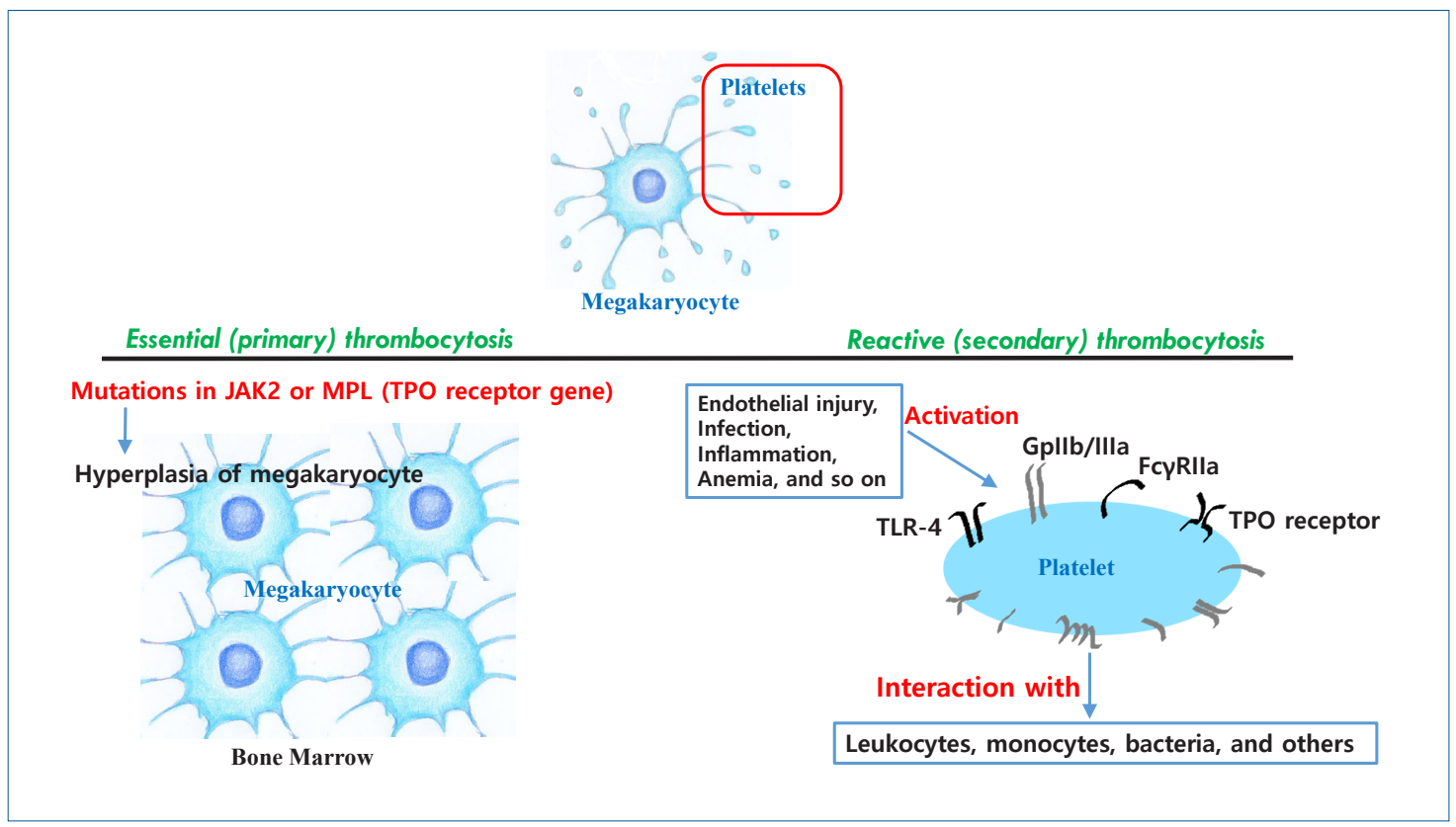

Graphical abstract. Thrombocytosis is frequently observed in newborn infants. Platelets, nonnucleated fragments arising from megakaryocytes, are activated by endothelial injury as well as infection, acute/chronic inflammation, and anemia. Activated platelets can interact with leukocytes, monocytes, and bacteria through multiple receptors. Reactive (secondary) thrombocytosis in newborn infants is benign and can spontaneously resolve. It is rarely associated with complications, unlike thrombocytosis in adults. Essential (primary) thrombocytosis, a myeloproliferative neoplasm, is rare in newborn infants and characterized by megakaryocytic hyperplasia in the bone marrow caused by mutations in the Janus kinase 2 (JAK2) or myeloproliferative leukemia (MPL) gene. TPO, thrombopoietin; TLR, Tall-like receptor.

the yolk sac to the liver and then to the bone marrow. Megakaryocytes are found in the yolk sac at a gestational age of 5 weeks and in the liver by a gestational age of 10 weeks, while platelets are first found in the circulation at a gestational age of 8-9 weeks. $^{6}$ ) Bone marrow megakaryopoiesis starts at a gestational age of 11 weeks and then dominates at a gestational age of 20 weeks. Thus, bone marrow is the primary site for megakaryopoiesis in newborn infants.

Megakaryopoiesis and platelet production (thrombopoiesis) are mainly regulated by TPO and other cytokines and hematopoietic growth factors such as interleukin-3 (IL-3), IL-6, and IL-11. ${ }^{7)}$ The increased production of these factors during infection or inflammation can stimulate megakaryocyte production, resulting in elevated platelet counts. Ishiguro et al. ${ }^{8)}$ reported that elevated serum TPO and IL- 6 concentrations precede thrombocytosis during acute infection. Platelets are activated by endothelial injury, infection, acute/chronic inflammation, and anemia. Following infection, platelets are activated to interact with leukocytes, monocytes, bacteria, and others through multiple receptors. Activated platelets can interact with bacteria through various receptors such as Tall-like receptor (TLR)-4, a low-affinity receptor for the constant fragment ( $\mathrm{Fc}$ ) of immunoglobulin G (FcyRIIa) and glycoprotein (Gp) IIb/IIIa.9) As a result, platelets assist in clearing bacteria by presenting them to neutrophils, thus enhancing phagocytosis during infection. ${ }^{7)}$

\section{Thrombopoietin}

The regulation of platelet counts relies on the negative feedback mechanism of TPO, which binds to the TPO receptor on platelets and megakaryocytes, reducing plasma-free TPO levels and decreasing thrombopoiesis. A reduction in platelet counts leads to elevated plasma-free TPO, resulting in increased thrombopoiesis. ${ }^{7}$ Platelets are mainly removed from the blood in the spleen and liver.

It is well established that TPO concentrations in newborn infants are higher than those in adults. ${ }^{10)}$ Levels increase after birth, peak on the 2 nd day of life, and start decreasing at 1 month of age, ${ }^{11)}$ and they are inversely correlated with platelet counts. Nakayama et al. ${ }^{12)}$ reported similar results that serum TPO levels soon after birth to 1 month of age are significantly higher than those at 2-6 months after birth. Thereafter, TPO levels decrease with age (Fig.1). ${ }^{13)}$ The low expression of TPO receptor on the platelets of newborn infants until 1 month of age decreases TPO clearance, leading to elevated plasma-free TPO levels and increased thrombopoiesis. ${ }^{12)}$ Megakaryocyte precursor cells of newborn infants are more sensitive to TPO than those of adults. ${ }^{2)}$ Platelet counts are typically low in small-for-gestationalage (SGA) newborns at birth. ${ }^{14)}$ However, TPO concentrations in SGA newborns remain controversial. TPO concentrations were significantly elevated in SGA newborns compared to appropriate-for-gestational-age newborns $(135 \mathrm{pg} / \mathrm{mL}$ vs. $94 \mathrm{pg} /$ $\mathrm{mL}$, respectively $)^{15)}$ and healthy term newborns $(86 \mathrm{pg} / \mathrm{mL}$ vs. 72 $\mathrm{pg} / \mathrm{mL}$, respectively). ${ }^{16)}$ On the other hand, TPO concentrations were not elevated in SGA newborns, especially those with liver problems. ${ }^{14}$

\section{Anemia and erythropoietin}

Erythropoietin (EPO), a regulator of erythrocyte production, 
is structurally similar to TPO with an amino terminal domain containing a sequence homologous to that of EPO. ${ }^{17,18)}$ Preterm infants with thrombocytosis show decreased red blood cell counts and elevated endogenous EPO levels due to anemia, which probably results in elevated platelet counts. ${ }^{4)}$ Increased production of thrombopoietic factors, cytokines, and other hematopoietic growth factors due to anemia might be related to elevated platelet counts as well..$^{2)}$ Yadav et al. ${ }^{19)}$ and Özcan et al. ${ }^{5)}$ reported that anemia was present in $29 \%$ and $9 \%$ of pediatric patients with thrombocytosis, respectively. It was also reported that $34 \%$ of newborn infants with anemia have thrombocytosis. ${ }^{20)}$ These findings support a negative feedback mechanism of endogenous EPO in thrombocytosis, which has functional as well as structural similarities to TPO. Recombinant human EPO, a potent thrombopoietic factor, also affects throm. bopoiesis and erythropoiesis. ${ }^{21,22)}$

\section{Infection and inflammation}

Platelets have proinflammatory activity in addition to procoagulant activity. Infectious pathogens and immune complexes formed by them can activate platelets, which then secrete molecules to activate immune reactions. ${ }^{23)}$ Thom et al. ${ }^{24)}$ sug. gested that rebound vigorous multilineage hematopoiesis following myelosuppression due to critical illnesses such as infection, inflammation, or iron deficiency can lead to hyperactive bone marrow and result in thrombocytosis.

\section{Classification of thrombocytosis}

\section{Classification by platelet count (Table 1)}

Thrombocytosis in newborn infants has variably been defined as a platelet count of $>400,000 / \mu \mathrm{L},>600,000 / \mu \mathrm{L}$, or $>900,000 /$ $\mu \mathrm{L}$. Thrombocytosis as defined and classified by Sutor ${ }^{25)}$ is wide-

Table 1. Classification by platelet count

\begin{tabular}{lc}
\hline Classification & Peak platelet count $(/ \mu \mathrm{L})$ \\
\hline Mild & $500,000-699,000$ \\
Moderate & $700,000-899,000$ \\
Severe & $900,000-999,000$ \\
Extreme & $\geq 1,000,000$ \\
\hline
\end{tabular}

ly used. Thrombocytosis has been defined as a platelet count $\geq 500,000 / \mu \mathrm{L}$ and classified as mild $(500,000-699,000 / \mu \mathrm{L})$, moderate $(700,000-899,000 / \mu \mathrm{L})$, severe $(900,000-999,000 / \mu \mathrm{L})$, or extreme $(\geq 1,000,000 / \mu \mathrm{L})$ according to peak platelet count. $\left.{ }^{26}\right)$

\section{Classification by etiology (Table 2)}

Neonatal thrombocytosis is also classified as essential (primary) or reactive (secondary). In most cases, this is a reactive process. Essential thrombocytosis, a myeloproliferative neoplasm with an estimated annual incidence rate of approximately 1.00 per 100,000 adults, is extremely rare in newborns. ${ }^{1)}$ The incidence of essential thrombocytosis in newborn infants is not well known. Essential thrombocytosis is represented by megakaryocytic hyperplasia in the bone marrow with thrombocytosis, which is mainly caused by mutations in Janus kinase 2 (JAK2) or myeloproliferative leukemia (MPL, a TPO receptor gene). ${ }^{27)}$ It is known to increase the risk of vascular events such as hemorrhage and thrombosis due to thrombocytosis. Antiplatelet or cytoreductive treatments are needed to reduce these complications. The differential diagnosis of essential thrombocytosis includes reactive (secondary) thrombocytosis, spurious thrombocytosis, or other forms of myeloproliferative neoplasms such as polycythemia vera or primary myelofibrosis. On the other hand, reactive thrombocytosis is considered a benign condition that is more common in newborns than in adults. Increased platelet production as a result of megakaryopoiesis occurs due to several conditions, such as infections, inflammation, tissue injury, and anemia, resulting in reactive thrombocytosis. Iron deficiency, hemorrhage, stress, exposure to epinephrine, vitamin E deficiency, and rebound from thrombocytopenia can also cause thrombocytosis. ${ }^{20)}$ Denton and Davis ${ }^{28)}$ reported that all pediatric cases of extreme thrombocytosis (platelet count, $\geq 1,000,000$ / $\mu \mathrm{L})$ are reactive, requiring no treatment. Wiedmeier et al. $\left.{ }^{26}\right)$ reported that all cases of extreme thrombocytosis (platelet count, $\geq 1,000,000 / \mu \mathrm{L}$ ) in newborns and young infants are reactive. All resolved without any hemorrhagic or thromboembolic compliations. They also reported that infection (mostly respiratory tract infections), postoperative status, and anemia could cause thrombocytosis, generally a benign and self-limiting condition. ${ }^{26}$ Reactive thrombocytosis rarely increases the risk of hemorrhagic and thromboembolic complications in newborn infants, and it usually does not require antiplatelet or cytoreductive treatment. ${ }^{29)}$

Table 2. Classification by etiology

\begin{tabular}{lll}
\hline Etiology & \multicolumn{1}{c}{ Essential (primary) } & Reactive (secondary) \\
\hline Incidences & Extremely rare in newborn infants & More common in newborn infants than in adults \\
Causes & $\begin{array}{c}\text { Mutations in JAK2 or MPL (TPO receptor gene) } \rightarrow \text { megakaryocytic } \\
\text { hyperplasia in the bone marrow }\end{array}$ & $\begin{array}{c}\text { Infection, acute/chronic inflammation, tissue injury, } \\
\text { anemia }\end{array}$ \\
Differential diagnosis & $\begin{array}{c}\text { Reactive (secondary) thrombocytosis } \\
\text { Other forms of myeloproliferative neoplasms (polycythemia vera or } \\
\text { primary myelofibrosis) }\end{array}$ & $\begin{array}{l}\text { Essential (primary) thrombocytosis } \\
\text { Treatments }\end{array}$ \\
$\begin{array}{lll}\text { Compliplatelet or cytoreductive treatment } \\
\text { Arterial thrombosis, myocardial infarction, hemorrhage, microvascular } \\
\text { occlusion in adults }\end{array}$ & Requiring no treatment for thrombocytosis \\
\hline
\end{tabular}

JAK2, Janus kinase 2; MPL, myeloproliferative leukemia; TPO, thrombopoietin. 
Elevated platelet counts have also been reported in infants with severe osteogenesis imperfecta, notably those younger than 2 years of age. The authors suggested that elevated platelet counts in infants with severe osteogenesis imperfecta can serve as a marker of inflammation. ${ }^{30)}$ Asplenia $^{2)}$ and sickle cell disease ${ }^{31)}$ can also cause thrombocytosis, especially cases of extreme thrombocytosis (platelet count, $\geq 1,000,000 / \mu \mathrm{L}$ ) due to reduced platelet storage and removal.

\section{Initial platelet count at birth by gestational age}

Platelet counts increase with advancing gestational age. The initial platelet count at birth is correlated with gestational age, which is increased by $2,089 / \mu \mathrm{L}$ as gestational age increases by 1 week. Thus, preterm infants have lower initial platelet counts than late-preterm or term infants (Fig. 2). ${ }^{11)}$ These findings are speculated to be related to higher TPO concentrations at birth in preterm infants than in term infants. ${ }^{3)}$ There is an inverse correlation between TPO concentrations and platelet counts due to the negative feedback mechanism of TPO.

\section{Platelet count by postnatal age}

Platelet counts increase with advancing postnatal day, indicating a positive correlation with postnatal age. According to Wiedmeier et al., ${ }^{11)}$ platelet counts are as high as $750,000 / \mu \mathrm{L}$ at 2-3 weeks and 6-7 weeks after birth. The cause of thrombocytosis at 2-3 weeks of age was speculated to be a neonatal surge in TPO. ${ }^{11)}$ The cause of thrombocytosis at 6-7 weeks was not apparent. It has been suggested that infection, hypoxemia, and anemia can temporarily elevate platelet counts. ${ }^{11)}$ According to Nakayama et al., ${ }^{12)}$ the initial platelet count in preterm infants

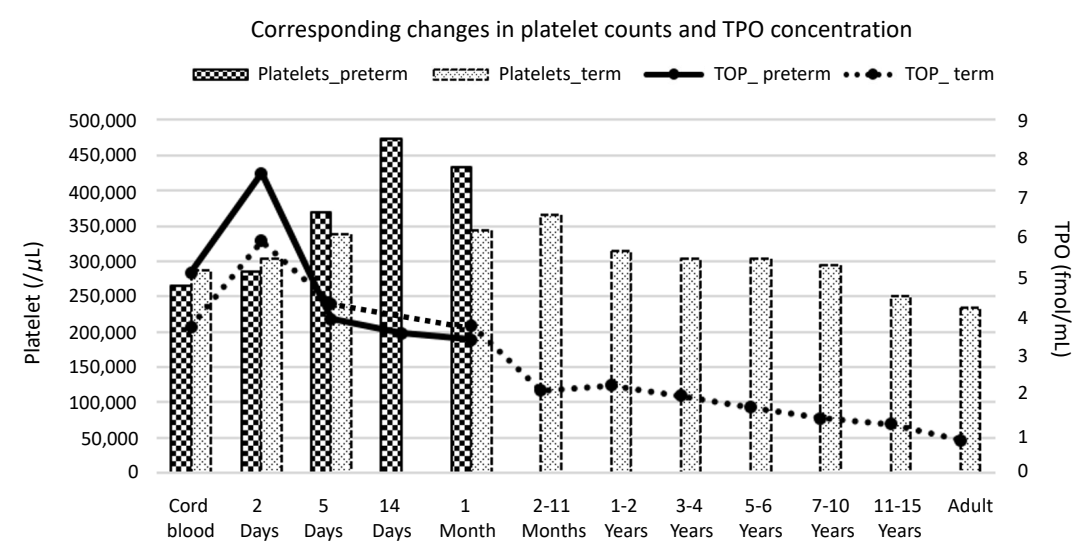

Fig. 1. Corresponding changes in platelet counts and thrombopoietin (TPO) concentration. TPO concentrations in newborn infants are increased after birth, peak on the 2 nd day after birth, and start to decrease at 1 month of age. TPO levels then decrease with age. We found an inverse correlation between TPO concentration and platelet count. Preterm infants had higher initial TPO concentrations and lower initial platelet counts than term infants. Thrombocytosis during the first few postnatal weeks is more common in preterm infants than in term infants. Plotted from the results of Matsubara et al. ${ }^{3)}$ and Ishiguro et al. ${ }^{13)}$

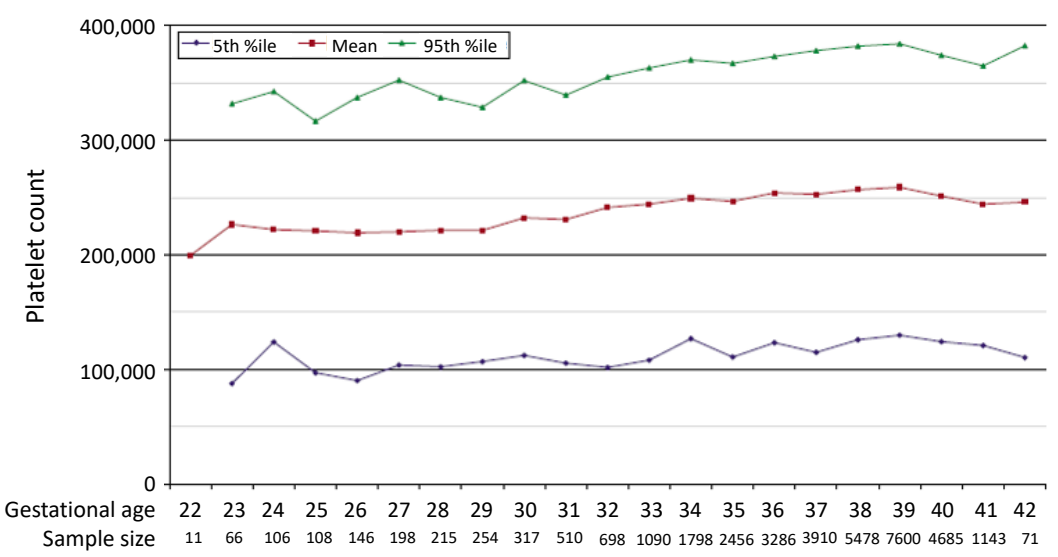

Fig. 2. Platelet counts by gestational age. Platelet counts are shown for neonates with a gestational age of 22-42 weeks. Platelet counts increase with advancing gestational age, which is increased by $2,089 / \mu \mathrm{L}$ as gestational age advanced by 1 week. Thus, preterm infants have lower initial platelet counts than term infants. The mean values and 5 th and 95 th percentiles are given. Adapted from Wiedmeier et al., ${ }^{11)}$ with permission of Springer Nature. 
at birth was $230,000 / \mu \mathrm{L}$, and it gradually increased to $579,000 /$ $\mu \mathrm{L} 5$ weeks of life. This increase continued, reaching a relatively high level at approximately 8 weeks of life.

\section{Thrombocytosis in preterm infants}

Thrombocytosis is more frequently observed in preterm infants than in term infants during the first few postnatal weeks to months. In one study, thrombocytosis was found in 38\% of low birth weight preterm infants. ${ }^{3)}$ Thrombocytosis was observed in $32.6 \%$ of preterm infants with a gestational age $<32$ weeks who had not received recombinant human EPO. ${ }^{4)}$ Higher TPO concentrations ${ }^{3,32)}$ and more sensitive megakaryocyte precursor $\mathrm{cells}^{3)}$ in preterm versus term infants are the speculated cause (Fig. 1). ${ }^{3)}$ Low expression of TPO receptor in preterm infants leads to the accumulation of free TPO, which has been suspected to induce thrombocytosis in preterm infants. ${ }^{12)}$ Anemia was frequently diagnosed in preterm infants with a gestational age $<$ 32 weeks by Del Rey Hurtado de Mendoza et al., ${ }^{4)}$ who reported an inverse correlation between decreased red blood cell counts and increased platelet counts in preterm infants.

Thrombocytosis was reportedly more common in preterm infants with a gestational age $<27$ weeks than in those with a gestational age $\geq 27$ weeks. ${ }^{20)}$ Thrombocytosis in preterm infants was most common at a postmenstrual age (PMA) of 35 weeks, indicating that postnatal platelet regulation would mature at a PMA of approximately 35 weeks. McPherson and Juul ${ }^{20)}$ suggested that the maturation of platelet regulation develops with PMA. The overall prevalence of abnormal platelet counts decreases with maturity in preterm infants. ${ }^{20)}$ Developmental immaturity of platelet regulation might be associated with frequent thrombocytosis in preterm infants.

\section{Complications of thrombocytosis}

Thrombocytosis can increase the risk of arterial thrombosis, myocardial infarction, hemorrhage, and microvascular occlusion in adults. ${ }^{1,2)}$ Moreover, adults with essential thrombocytosis are at an increased risk of leukemic transformation or evolution to myelofibrosis. ${ }^{33)}$ However, reactive thrombocytosis, notably extreme thrombocytosis, in newborn infants does not seem to be associated with an increased risk of hemorrhagic or thromboembolic complications. ${ }^{24,26,28,29)}$ Preterm infants with a gestational age $<32$ weeks and thrombocytosis had a higher incidence of retinopathy of prematurity according to Del Rey Hurtado de Mendoza et al. ${ }^{4)}$ Conversely, other studies reported that thrombocytopenia, not thrombocytosis, is a risk factor for retinopathy of prematurity. ${ }^{34)}$ Thus, the association between thrombocytosis and retinopathy of prematurity remains controversial.

\section{Conclusion}

Thrombocytosis is frequently found in newborns and young infants, especially those younger than 2 years of age. We often encounter preterm infants with thrombocytosis in neonatal intensive care units. Initial platelet counts at birth increase with advancing gestational age. Preterm infants have lower initial platelet counts than late-preterm or term infants. Thrombocytosis during the first few postnatal weeks to months is more frequently observed in preterm than term infants. A high TPO concentration and low TPO receptor expression on platelets leading to elevated plasma-free TPO levels, more sensitive megakaryocyte precursor cells to TPO, decreased red blood cell counts, and immaturity of platelet regulation have been speculated to cause thrombocytosis in preterm infants. Thrombocytosis in newborn infants, a reactive process following infection, acute/chronic inflammation, or anemia, is benign and resolves spontaneously, and unlike in adults, rarely associated with hemorrhagic or thromboembolic complications.

\section{Footnotes}

Conflict of interest: No potential conflict of interest relevant to this article was reported.

Funding: This study received no specific grant from any funding agency in the public, commercial, or not-for-profit sectors.

ORCID:

Ga Won Jeon https://orcid.org/0000-0002-8206-9727

\section{References}

1. Jensen MK, de Nully Brown P, Nielsen OJ, Hasselbalch HC. Incidence, clinical features and outcome of essential thrombocythaemia in a well defined geographical area. Eur J Haematol 2000;65:132-9.

2. Dame C, Sutor AH. Primary and secondary thrombocytosis in childhood. Br J Haematol 2005; 129:165-77.

3. Matsubara K, Baba K, Nigami H, Harigaya H, Ishiguro A, Kato T, et al. Early elevation of serum thrombopoietin levels and subsequent thrombocytosis in healthy preterm infants. Br J Haematol 2001;115:9638.

4. Del Rey Hurtado de Mendoza B, Esponera CB, Izquierdo Renau M, Iglesias Platas I. Asymptomatic late thrombocytosis is a common finding in very preterm infants even in the absence of erythropoietin treatment. J Int Med Res 2019;47:1504-11.

5. Özcan C, Şaylı TR, Koşan-Çulha V. Reactive thrombocytosis in children. Turk J Pediatr 2013;55:411-6.

6. Davenport P, Liu ZJ, Sola-Visner M. Changes in megakaryopoiesis over ontogeny and their implications in health and disease. Platelets 2020; 31:692-9.

7. Margraf A, Zarbock A. Platelets in inflammation and resolution. J Immunol 2019;203:2357-67.

8. Ishiguro A, Suzuki Y, Mito M, Shimbo T, Matsubara K, Kato T, et al. Elevation of serum thrombopoietin precedes thrombocytosis in acute infections. Br J Haematol 2002;116:612-8.

9. Fitzgerald JR, Foster TJ, Cox D. The interaction of bacterial pathogens 
with platelets. Nat Rev Microbiol 2006;4:445-57.

10. Sola-Visner M, Sallmon H, Brown R. New insights into the mechanisms of nonimmune thrombocytopenia in neonates. Semin Perinatol 2009;33: 43-51.

11. Wiedmeier SE, Henry E, Sola-Visner MC, Christensen RD. Platelet reference ranges for neonates, defined using data from over 47,000 patients in a multihospital healthcare system. J Perinatol 2009;29:130-6.

12. Nakayama H, Ihara K, Hikino S, Yamamoto J, Nagatomo T, Takemoto $\mathrm{M}$, et al. Thrombocytosis in preterm infants: a possible involvement of thrombopoietin receptor gene expression. J Mol Med (Berl) 2005;83: 316-20.

13. Ishiguro A, Nakahata T, Matsubara K, Hayashi Y, Kato T, Suzuki Y, et al. Age-related changes in thrombopoietin in children: reference interval for serum thrombopoietin levels. Br J Haematol 1999;106:884-8.

14. Maruyama H, Nakamura M, Yonemoto N, Kageyama M. Thrombocytopenia at birth is a predictor of cholestasis in infants with small for gestational age. Acta Med Okayama 2013;67:219-25.

15. Amarilyo G, Oren A, Mimouni FB, Ochshorn Y, Deutsch V, Mandel D. Increased cord serum inflammatory markers in small-for-gestational-age neonates. J Perinatol 2011;31:30-2.

16. Wasiluk A, Mantur M, Kemona H, Szczepański M, Jasińska E, Milewski R. Thrombopoiesis in small for gestational age newborns. Platelets 2009; 20:520-4.

17. Gutti U, Pasupuleti SR, Sahu I, Kotipalli A, Undi RB, Kandi R, et al. Erythropoietin and thrombopoietin mimetics: Natural alternatives to erythrocyte and platelet disorders. Crit Rev Oncol Hematol 2016;108: 175-86.

18. Gurney AL, Kuang WJ, Xie MH, Malloy BE, Eaton DL, de Sauvage FJ. Genomic structure, chromosomal localization, and conserved alternative splice forms of thrombopoietin. Blood 1995;85:981-8.

19. Yadav D, Chandra J, Sharma S, Singh V. Clinicohematological study of thrombocytosis. Indian J Pediatr 2010;77:643-7.

20. McPherson RJ, Juul S. Patterns of thrombocytosis and thrombocytopenia in hospitalized neonates. J Perinatol 2005;25:166-72.

21. Haiden N, Cardona F, Schwindt J, Berger A, Kuhle S, Homoncik M, et al. Changes in thrombopoiesis and platelet reactivity in extremely low birth weight infants undergoing erythropoietin therapy for treatment of anaemia of prematurity. Thromb Haemost 2005;93:118-23.

22. Stohlawetz PJ, Dzirlo L, Hergovich N, Lackner E, Mensik C, Eichler HG, et al. Effects of erythropoietin on platelet reactivity and thrombopoiesis in humans. Blood 2000;95:2983-9.

23. Garraud O, Hamzeh-Cognasse H, Pozzetto B, Cavaillon JM, Cognasse F. Bench-to-bedside review: platelets and active immune functions - new clues for immunopathology? Crit Care 2013;17:236.

24. Thom CS, Echevarria E, Osborne AD, Carr L, Rubey KM, Salazar E, et al. Extreme thrombocytosis is associated with critical illness and young age, but not increased thrombotic risk, in hospitalized pediatric patients. J Thromb Haemost 2020;18:3352-8.

25. Sutor AH. Thrombocytosis in childhood. Semin Thromb Hemost 1995; 21:330-9.

26. Wiedmeier SE, Henry E, Burnett J, Anderson T, Christensen RD. Thrombocytosis in neonates and young infants: a report of 25 patients with platelet counts of $>$ or $=1000000$ microl(-1.. J Perinatol 2010;30: 222-6.

27. Lee JM, Lee H, Eom KS, Lee SE, Kim M, Kim Y. Impact of integrated genetic information on diagnosis and prognostication for myeloproliferative neoplasms in the next-generation sequencing era. J Clin Med 2021;10:1033.

28. Denton A, Davis P. Extreme thrombocytosis in admissions to paediatric intensive care: no requirement for treatment. Arch Dis Child 2007;92: 515-6.

29. Christensen RD, Henry E, Del Vecchio A. Thrombocytosis and thrombocytopenia in the NICU: incidence, mechanisms and treatments. J Matern Fetal Neonatal Med 2012;25 Suppl 4:15-7.

30. Salter L, Offiah AC, Bishop N. Elevated platelet counts in a cohort of children with moderate-severe osteogenesis imperfecta suggest that inflammation is present. Arch Dis Child 2018;103:767-71.

31. Brousse V, Buffet P, Rees D. The spleen and sickle cell disease: the sick(led. spleen. Br J Haematol 2014;166:165-76.

32. Albert TS, Meng YG, Simms P, Cohen RL, Phibbs RH. Thrombopoietin in the thrombocytopenic term and preterm newborn. Pediatrics 2000;105: 1286-91.

33. Lim Y, Lee JO, Bang SM. Incidence, survival and prevalence statistics of classical myeloproliferative neoplasm in Korea. J Korean Med Sci 2016;31:1579-85.

34. Vinekar A, Hegde K, Gilbert C, Braganza S, Pradeep M, Shetty R, et al. Do platelets have a role in the pathogenesis of aggressive posterior retinopathy of prematurity? Retina 2010;30:S20-3.

How to cite this article: Jeon GW. Pathophysiology, classification, and complications of common asymptomatic thrombocytosis in newborn infants. Clin Exp Pediatr 2022;65:182-7. https://doi.org/10.3345/cep.2021.00864 